\title{
Transcranial MR-guided focused ultrasound sonothrombolysis in the treatment of intracerebral hemorrhage
}

\author{
Stephen J. Monteith, M.D., ${ }^{1}$ Neal F. Kassell, M.D., ${ }^{1}$ Oded Goren, M.D., ${ }^{2}$ \\ and SAGi HaRnof, M.D. ${ }^{2}$ \\ ${ }^{1}$ Department of Neurosurgery, University of Virginia Health System, Charlottesville, Virginia; \\ and ${ }^{2}$ Department of Neurosurgery, Sheba Medical Center, Tel Hashomer, Israel
}

\begin{abstract}
Intracerebral hemorrhage remains a significant cause of morbidity and mortality. Current surgical therapies aim to use a minimally invasive approach to remove as much of the clot as possible without causing undue disruption to surrounding neural structures. Transcranial MR-guided focused ultrasound (MRgFUS) surgery is an emerging technology that permits a highly concentrated focal point of ultrasound energy to be deposited to a target deep within the brain without an incision or craniotomy. With appropriate ultrasound parameters it has been shown that MRgFUS can effectively liquefy large-volume blood clots through the human calvaria. In this review the authors discuss the rationale for using MRgFUS to noninvasively liquefy intracerebral hemorrhage (ICH), thereby permitting minimally invasive aspiration of the liquefied clot via a small drainage tube. The mechanism of action of MRgFUS sonothrombolysis; current investigational work with in vitro, in vivo, and cadaveric models of ICH; and the potential clinical application of this disruptive technology for the treatment of ICH are discussed.

(http://thejns.org/doi/abs/10.3171/2013.2.FOCUS1313)
\end{abstract}

KeY WordS • intracerebral hemorrhage • MRgFUS • sonothrombolysis • focused ultrasound $\bullet$ high-intensity focused ultrasound

I NTRACEREBRAL hemorrhage accounts for $10 \%-15 \%$ of the approximately 15 million strokes that occur worldwide every year. The annual incidence is 10-30 per 100,000 and is the cause of significant morbidity and mortality. ${ }^{20,34,35,40}$ The 30-day mortality rate is on the order of $35 \%-52 \%$, with half of those deaths occurring in the first 2 days. ${ }^{4}$ The neurological deficit caused by ICH is multifactorial. The mass effect from the clot causes mechanical disruption of brain tissue and an increase in intracranial pressure. There is surrounding edema with associated decreased cerebral blood flow, as well as release of toxic metabolites and free radical generation from breakdown of blood products. Ischemia ensues, cell metabolism breaks down, and cells swell and die, resulting in further neurological deficit after the initial insult.

The clot volume and clinical status of the patient has clearly been shown to be a valuable predictor of outcome. Broderick et al. ${ }^{1}$ demonstrated that hemorrhage volume (0-29 $\mathrm{cm}^{3}, 30-60 \mathrm{~cm}^{3}$, and $61 \mathrm{~cm}^{3}$ or more), calculated by an ellipsoid method $(4 / 3 \times \pi \mathrm{abc}$, where $\mathrm{a}, \mathrm{b}$, and $\mathrm{c}$ are the

\footnotetext{
Abbreviations used in this paper: $\mathrm{ICH}=$ intracerebral hemorrhage; IVH = intraventricular hemorrhage; MRgFUS = MR-guided focused ultrasound; tPA = tissue plasminogen activator.
}

3 dimensions of the clot) and 2 categories of the Glasgow Coma Scale score ( 9 or more and 8 or less), 30 -day mortality could be predicted. Hemorrhage volume of $60 \mathrm{~cm}^{3}$ or more on the initial CT scan and a Glasgow Coma Scale score of 8 or less had a predicted 30-day mortality of $91 \%$. Patients with a volume of less than $30 \mathrm{~cm}^{3}$ and a Glasgow Coma Scale score of 9 or more had a predicted 30-day mortality of $19 \%$.

Clinical symptoms can worsen due to hematoma enlargement and edema. Hematoma enlargement generally occurs within 12 hours after symptom onset, most frequently during the first 3 hours. ${ }^{2,19}$ Perihematomal edema peaks around 5-6 days after hemorrhage and lasts up to 14 days. Most (about $75 \%$ by volume) of the perihematomal edema occurs in the first 24 hours. ${ }^{3,10,15}$ A large edema volume has been found to be the greatest contributor to worse clinical outcomes. ${ }^{11}$ Decreasing this edema volume as soon as possible is the basis for early intervention to remove the ICH. ${ }^{24,25,33}$ Further rationale for removal of the $\mathrm{ICH}$ is that if the $\mathrm{ICH}$ is large in addition to significant edema, the intracranial pressure will be high. As a result the cerebral perfusion pressure is compromised leading to ischemia, particularly in watershed zones due to decreased perfusion. This increases neurological injury further by enlarging the area of cell death. 


\section{The Need for Minimally Invasive Therapies}

The role of surgical intervention for the treatment of spontaneous ICH is unclear. Current management generally consists of supportive care with reversal of coagulopathy, management of hypertension, and placement of an external ventricular drain in patients with hydrocephalus. Results of the STICH (International Surgical Trial in Intracerebral Haemorrhage) demonstrated no benefit in surgical evacuation versus conservative management. ${ }^{24}$ Subgroup analysis of the 223 patients with lobar ICH and no IVH demonstrated a trend toward improved outcome with early surgery; however, the study was not powered sufficiently to delineate this finding clearly. ${ }^{24} \mathrm{~A}$ metaanalysis of 12 prospective randomized controlled trials was performed by Mendelow et al. ${ }^{25}$ Their results demonstrated improved outcomes for surgery performed for lobar hemorrhage in terms of both death (OR 0.8, 95\% CI 0.71-1.02) and death and severe disability (OR 0.86, 95\% CI 0.72-1.03). Current studies are underway to further delineate which patients will benefit from certain procedures. STICH II will investigate if surgery benefits patients with lobar ICH with clots that reach $1 \mathrm{~cm}$ of the cortical surface. Similarly, IVH with clot size no greater than $30 \mathrm{ml}$ is being investigated by the CLEAR-IVH (Clot Lysis Evaluating Accelerated Resolution of IVH) trial. ${ }^{30}$

Minimally invasive clot evacuation surgery for ICH with the goal of minimal brain disruption has been described. ${ }^{26}$ The use of tPA to accelerate clot dissolution has also been performed and shows increased rates of clot reduction compared with controls. The authors concluded in this preliminary report that there was a tentative indication that minimally invasive surgery plus recombinant tPA showed improved clot resolution versus traditional conservative management. ${ }^{31}$ The MISTIE II (Minimally Invasive Surgery plus tPA for Intracranial Hemorrhage Evacuation) trial conducted from 2006 to 2011 examined the use of recombinant tPA combined with minimally invasive surgery. Preliminary results were presented at the International Stroke Conference in New Orleans in February 2012 (http://braininjuryoutcomes.com/studies/mistie/ entry/mistie/international-stroke-conference-2012-mistiephase-2-results). The findings demonstrated a trend toward improved outcomes in patients treated, particularly if more hematoma was removed. Careful placement of the aspiration catheter along the long axis of the clot was noted to be an important factor in clot evacuation. A Phase III study (MISTIE III) is currently being designed with sufficient power to determine which patients will benefit the most from this technique.

Clot lysis combined with tPA has been shown to have increased efficiency when combined with ultrasound. Newell et al. ${ }^{33}$ used the EKOS ultrasound device in combination with a standard drainage catheter and tPA. Recombinant tPA was delivered directly into spontaneous $\mathrm{ICH}$ or IVH in 9 patients. A ventricular drainage catheter and the EKOS catheter were stereotactically implanted in the clot, and recombinant tPA and ultrasound were delivered for 24 hours. The mean percentage in volume reduction after 24 hours of therapy was $59 \%$ for $\mathrm{ICH}$ and
$45 \%$ for IVH. Clinical improvements were seen in 7 of 9 patients, with 1 death within 30 days. ${ }^{33}$

\section{Background of Transcranial MRgFUS}

The overall principle of MRgFUS therapy is combining 2 innovative technologies: the highly detailed images from MRI, and the therapeutic capabilities afforded by modern phased-array ultrasound transducers. The history and development of transcranial MRgFUS as a treatment modality has been published previously. ${ }^{16,23}$ The basic principle is that a large number of ultrasound beams pass harmlessly through the brain where they eventually focus accurately onto a therapeutic target, with the ultrasound energy combining to create a dramatic effect at the target. Cooled, degassed water circulates around the patient's scalp and is kept in place by a diaphragm placed around the patient's head to keep the water in. This allows ultrasound passage from the transducer to the patient. Depending on the ultrasound parameters delivered, a wide range of therapeutic effects can be achieved-from thermal ablation $^{7,18}$ and sonothrombolysis ${ }^{29}$ to temporary opening the blood-brain barrier for the targeted delivery of chemotherapeutics. ${ }^{22,42}$ Initially the defocusing effect of the irregular thickness of the skull bone prevented treatment of intracranial lesions through an intact skull. For this reason, in the first human clinical trials a craniectomy was performed to create an acoustic window. ${ }^{12,36}$ In light of this Ram et al. ${ }^{36}$ treated patients with glioblastoma via a craniectomy window. Low-frequency phased array transducers and phase correction based on CT scanning of the skull have provided solutions to the problems posed by the skull. ${ }^{14,41}$ A clinical trial for the transcranial treatment of patients with high-grade gliomas is ongoing in Boston. ${ }^{13}$ A similar system was used in Zurich in which 10 patients were treated transcranially with thalamotomies for chronic pain. ${ }^{21,32}$ Elias et al. ${ }^{7}$ from the University of Virginia have treated patients with essential tremor using the transcranial system with encouraging results. Similarly, Jeanmonod et al. ${ }^{17}$ have used MRgFUS in patients with Parkinson disease with a similar system and have achieved tremor control without any significant adverse events. Multicenter clinical trials using MRgFUS for metastatic brain tumors and glioblastoma are ongoing.

\section{Sonothrombolysis-Mechanism of Action}

Until now, transcranial MRgFUS use in patients has used thermal ablation by tuning a transcranial system (InSightec Neuro $650 \mathrm{kHz}$ ) to deliberately heat tissue at the focal point. ${ }^{7,17,18}$ In the setting of ultrasound sonothrombolysis for $\mathrm{ICH}$, increasing temperature is unwanted. The role of heat increasing the rate of sonothrombolysis with focused ultrasound has not been shown to be significant, and the process is largely mechanical rather than thermal. ${ }^{8,39}$ In the case of sonothrombolysis with fibrinolytic adjuncts such as tPA, microstreaming occurs with stable cavitation (oscillation in the size of microbubbles undergoing ultrasound delivery). This effect was used in the patients treated by Newell et al. ${ }^{33}$ using the EKOS ultrasound device and tPA. This process facilitates the passage of fibrinolytic 
through the clot, thereby acting on a fibrin network that is already under mechanical stress from the ultrasound energy. This synergistic effect improves the efficiency of the lysis process. ${ }^{5,9}$ In the setting of sonothrombolysis without fibrinolytics, inertial cavitation (oscillation is the size of microbubbles undergoing ultrasound delivery with subsequent violent collapse) is believed to be the major contributor to clot lysis. This process occurs with less control and predictability, with potential for inertial cavitation at sites distant to the target, thereby raising safety concerns..$^{38}$ After MRgFUS sonothrombolysis, the thin lysate is the color of motor oil and never reclots. Analysis of the lysate appears benign. ${ }^{6,27}$

\section{Current State of Experimental Data}

Initial studies by Harnof et al. showed that it was possible to use the transcranial MRgFUS system to liquefy clotted blood through an explanted human skull under MRI guidance (Fig. 1A and B) (Harnof S, presented at the Annual Conference for MRI Guided Focused Ultrasound Surgery, 2008; http://www.sendspace.com/pro/dl/0v9t8p). Subsequent experiments in swine by Harnof et al. demonstrated that preformed clots implanted into the brains of swine could be lysed without causing damage to the surrounding neural structures (Fig. 1C and D). Ultrasound parameter optimization using a $230-\mathrm{kHz} 1024$-element hemispheric phased array system was performed using human blood clots and an explanted human calvaria. ${ }^{29}$ The accuracy of the system is demonstrated in Fig. 2, with predictable volumes of lysis created in large-volume blood clots through an ex vivo human skull at locations delineated by the treatment plan. Increased $\mathrm{T} 2$ signal on MRI clearly demonstrates areas of lysis (Fig. 2). ${ }^{6}$ Largevolume intraventricular/intracerebral hemorrhages were subsequently created in cadavers, and transcranial noninvasive MRgFUS lysis was performed with precision accuracy (Fig. 3) (ultrasound parameters: frequency $230 \mathrm{kHz}$, $3950 \mathrm{~W}, 30$ seconds, $1-\mathrm{kHz}$ pulse repetition rate, $10 \%$ duty cycle). ${ }^{29}$ Liquefied clots in the cadaveric studies were able to be easily aspirated using a minimally invasive aspiration technique (1-cm incision, twist drill craniostomy hole, 2.1-mm aspiration tube) under MRI guidance, resulting in minimal residual $\mathrm{ICH}$ on postoperative imaging (Fig. 4). ${ }^{29}$ In vivo studies in swine with and without implanted ICH demonstrated the ultrasound process to be safe. Of note there was no damage to the brain surrounding the sonicated $\mathrm{ICH}$, and there was no permanent blood-brain barrier disruption on Gd-enhanced MRI or on histology after injection of Evans blue dye. There was no evidence of thermal necrosis on T2-weighted imaging or FLAIR MRI (immediate or 48 hours posttreatment) or on histology (Fig. 5). ${ }^{29}$

Because the ultrasound device parameters can be modified with the system's computer software during the procedure without moving the patient or the ultrasound transducer, there is the possibility of multimodal treatment of ICH/IVH using MRgFUS. For example, in the case of a casted ventricle, an MRgFUS cavitary lesion could be deliberately created in the septum pellucidum to enhance CSF circulation between the lateral ventricles, enhance
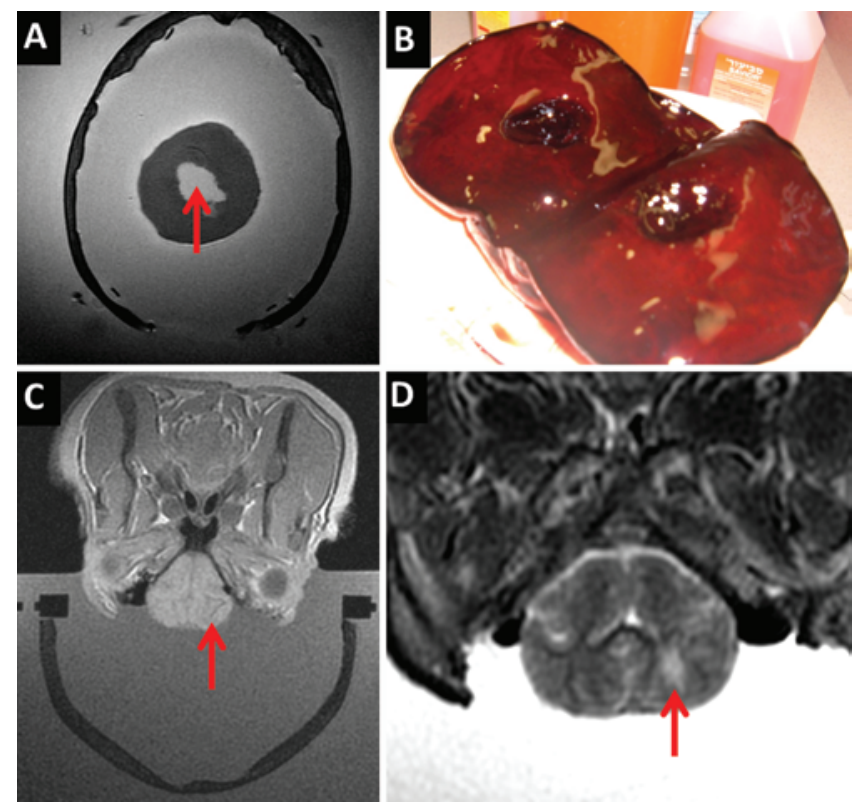

Fig. 1. A: Transcranial MRgFUS sonothrombolysis through an explanted human calvaria (axial section). The increased T2-weighted MR signal in the center of the clot (dark on T2) indicates lysis (arrow) after ultrasound sonications with a $230-\mathrm{kHz}$ hemispheric phased array transducer with CT correction for the aberration caused by the skull. B: Gross specimen of clotted blood removed from the explanted human calvaria in panel A. Areas of lysis corresponding to the area of lysis on MRI can be clearly seen. C: A formed clot is created in vitro and is surgically implanted as 1 piece into the swine brain (arrow). The clot is visible with gradient echo MRI. Transcranial MRgFUS sonothrombolysis is performed through an explanted human calvaria (seen in coronal section) using CT correction. D: Postsonothrombolysis T2-weighted MR image demonstrating lysis of the implanted $\mathrm{ICH}$ (arrow) by an increase in T2-weighted MR signal. There is no additional injury to the surrounding brain parenchyma as a result of the ultrasound treatment.
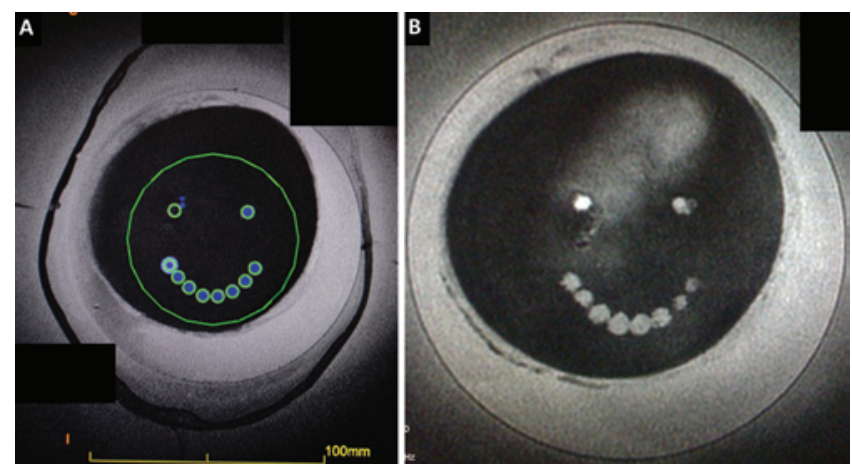

Fig. 2. Transcranial MRgFUS in an in vitro setup. A: Using an ex vivo human skull, a large blood clot is placed inside the skull and a treatment plan is created. Each green circle represents a treatment spot or sonication in the clot. The large green circle represents the treatment envelope and is the area in which sonications can be performed successfully. The treatment envelope can be expanded intraoperatively by moving the ultrasound transducer if required. B: Transcranial MRgFUS sonothrombolysis is performed under real-time MR guidance with precision accuracy. Lysis of the clot is visible as an increased T2weighted signal as per the treatment plan (frequency $230 \mathrm{kHz}$, power $3950 \mathrm{~W}$, duty cycle $10 \%$, sonication duration 30 seconds). Ultrasound parameter optimization with in vitro testing was performed prior to successfully liquefying the $\mathrm{ICH} / \mathrm{IVH}$ in a newly developed cadaveric model of $\mathrm{ICH} / \mathrm{IVH}$, and in an in vivo $\mathrm{ICH}$ model in swine. 

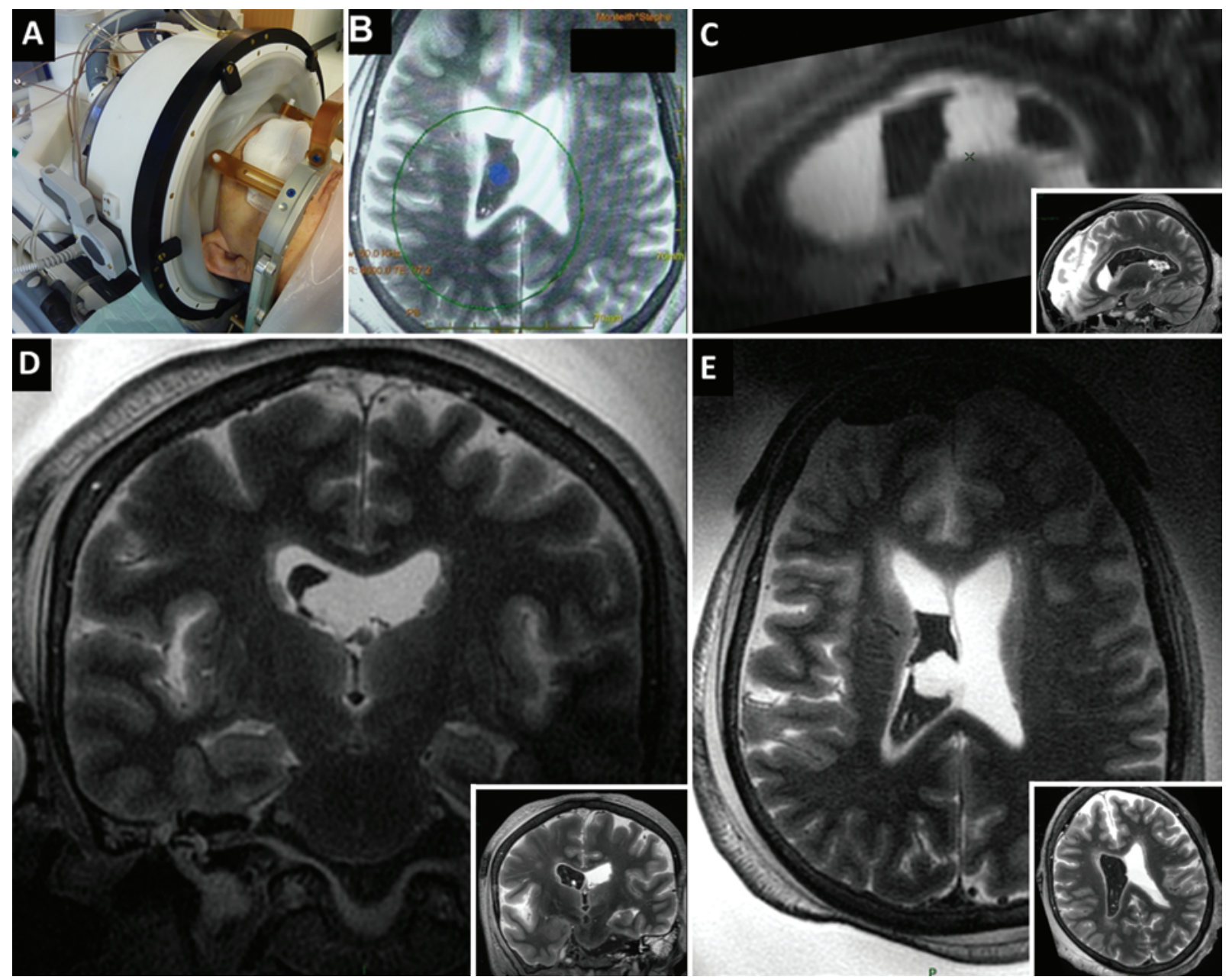

FIG. 3. Transcranial MRgFUS sonothrombolysis of an IVH after the first sonication. A: The cadaver is placed in the stereotactic frame, and the transducer is filled with circulating degassed water. B: The blue circle represents the treatment plan for the first sonication to be made in the middle of the IVH. The green circle represents the treatment envelope and can be expanded intraoperatively by moving the transducer. C-E: Sagittal, coronal, and axial T2-weighted MR images obtained before (insets) and after one sonication, clearly demonstrating increased T2 signal caused by incisionless MRgFUS sonothrombolysis. Printed with permission from Monteith et al: J Neurosurg 118:1035-1045, 2013.

clot dissolution, and improve hydrocephalus. In addition, treating hydrocephalus from an IVH via an incisionless third ventriculostomy or fenestration of the lamina terminalis may be possible due to the MR-guided accuracy and rapid energy drop achieved with MRgFUS. Thus far such procedures have been performed in cadaveric feasibility studies but may be of added utility in the future. ${ }^{28}$

\section{MRgFUS Treatment of ICH}

MRgFUS sonothrombolysis may facilitate a highly controlled, minimally invasive method to remove an ICH. The procedure can be performed in one sitting with immediate aspiration of the lysate, thereby removing the requirement for indwelling catheters or tubes in the brain that may harbor complications such as infection, malfunction, or breakage..$^{33}$ There is no requirement for tPA, which may worsen edema. ${ }^{37}$ The risk of hemorrhage down the catheter tract is also decreased if no tPA is needed. The lack of tPA may make the procedure safer because with
tPA the distribution of the lytic drug is less controlled and may lead to rebleeding at the site of the ICH. ${ }^{31}$ The targeting of MRgFUS is extremely precise, ${ }^{32}$ and intraoperative MRI provides real-time feedback on clot lysis as demonstrated by increased T2-weighted signal change. ${ }^{6}$ Ongoing monitoring with MRI as the lysis process progresses gives the surgeon the ability to determine when to stop and perform the aspiration procedure. One of the issues with current catheter-based approaches is that the catheter needs to be placed along the axis of the clot for maximal dissolution. ${ }^{31}$ It may be difficult or impossible to get the perfect trajectory through the long axis of the clot, without passing the catheter through critical structures. The shape of the clot is not of concern using the MRgFUS technique as the clot can be targeted using a precise treatment plan. Due to the minimally invasive nature of the procedure, repeated or staged transcranial sonothrombolysis procedures could be performed if required.

The high initial setup cost for the MRgFUS system and associated 3-T MRI scanner may be prohibitive; how- 


\section{Transcranial MRgFUS sonothrombolysis for ICH}

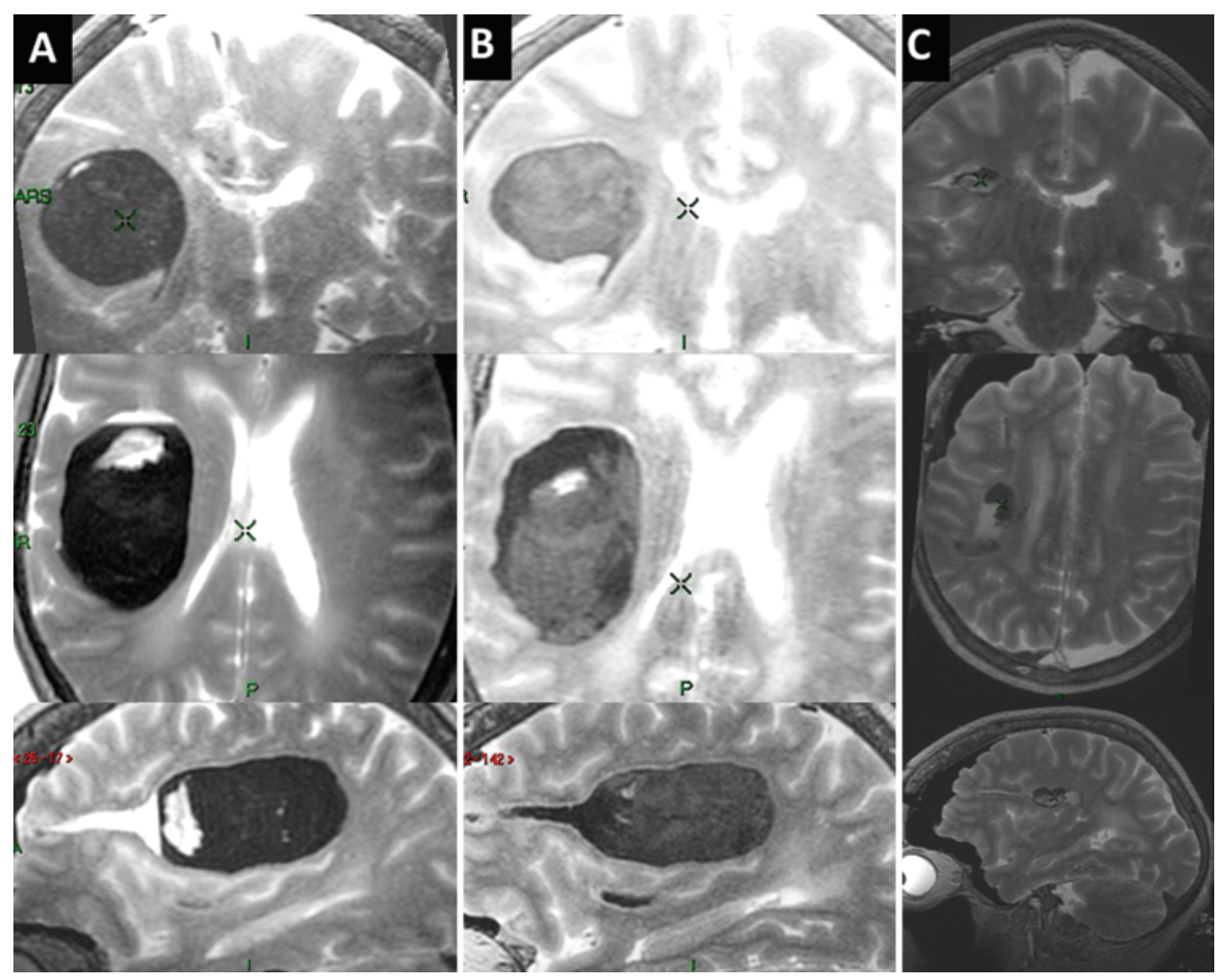

FIG. 4. Incisionless transcranial MRgFUS sonothrombolysis followed by minimally invasive aspiration of the ICH. A: Preoperative T2-weighted coronal (upper), axial (center), and sagittal (lower) MR images demonstrating a solid dark ICH in the right basal ganglia. B: Lysis of the ICH after transcranial MRgFUS is demonstrated by an increase in T2 signal in the targeted area. C: Minimal residual ICH (not targeted with MRgFUS) with improved mass effect on T2-weighted MR images. Liquid ICH evacuation was performed using a 1-cm incision, twist drill craniostomy, and MRI-guided aspiration via a 2.1-mm-diameter drainage tube. The use of tPA or a long-term indwelling drainage catheter is not necessary using this technique.

ever, it is likely the focused ultrasound system would be purchased for use with other neurosurgical applications in mind and not specifically for ICH. When not in use the MRI scanner can be used as a regular diagnostic device. Currently, additional staff members are required to support treatments with the MRgFUS system (ultrasound engineer and MRI technician). There is a need to shave the head, although this problem may be overcome with technical advances or the use of ultrasound compatible liquids/gels. The current treatment envelope of the transducer means that clots within approximately $2 \mathrm{~cm}$ to the skull surface are unable to be targeted due to current beam steering limitations.

The design of a clinical trial to test the feasibility and safety using MRgFUS for the treatment of ICH is currently underway. The patients most likely to benefit from MRgFUS treatment will only be determined from experience with a clinical trial; however, it is likely that patients with deep, central hemorrhages or IVH may benefit most as these lesions are in the sweet spot for the transducer and require less electrical steering of the ultrasound waves and therefore lysis efficiency is optimized. In terms of workflow, ideally a patient with ICH is diagnosed in the emergency department after routine CT scanning. The patient is transferred to the MRgFUS suite for immediate incisionless MRgFUS sonothrombolysis followed by MRguided aspiration using a small aspiration catheter and a minimal incision. Intraoperative and postoperative MRI demonstrates the degree of clot removal and evidence of potential complications.

\section{Conclusions}

MRgFUS sonothrombolysis for ICH is a technique in its infancy, and as such there are subtleties in the technical aspects that can only be learned with experience. This necessitates having neurosurgeons who are experienced in transcranial focused ultrasound therapy so that they are able to recognize potential complications from the focused ultrasound procedure and perform traditional emergency neurosurgical procedures if the situation requires it. A collaborative effort, from industry, neurosurgeons, radiologists, anesthesiologists, and support staff, is essential to push this potentially disruptive technology forward. Minimally invasive treatment of ICH with MRgFUS has been demonstrated with efficacy and safety in laboratory and in vivo models of ICH. The next step is evaluating the potential of this technique in the setting of a pilot clinical trial.

\section{Disclosure}

Dr. Kassell has ownership in InSightec.

Author contributions to the study and manuscript preparation include the following. Conception and design: Monteith, Harnof. 

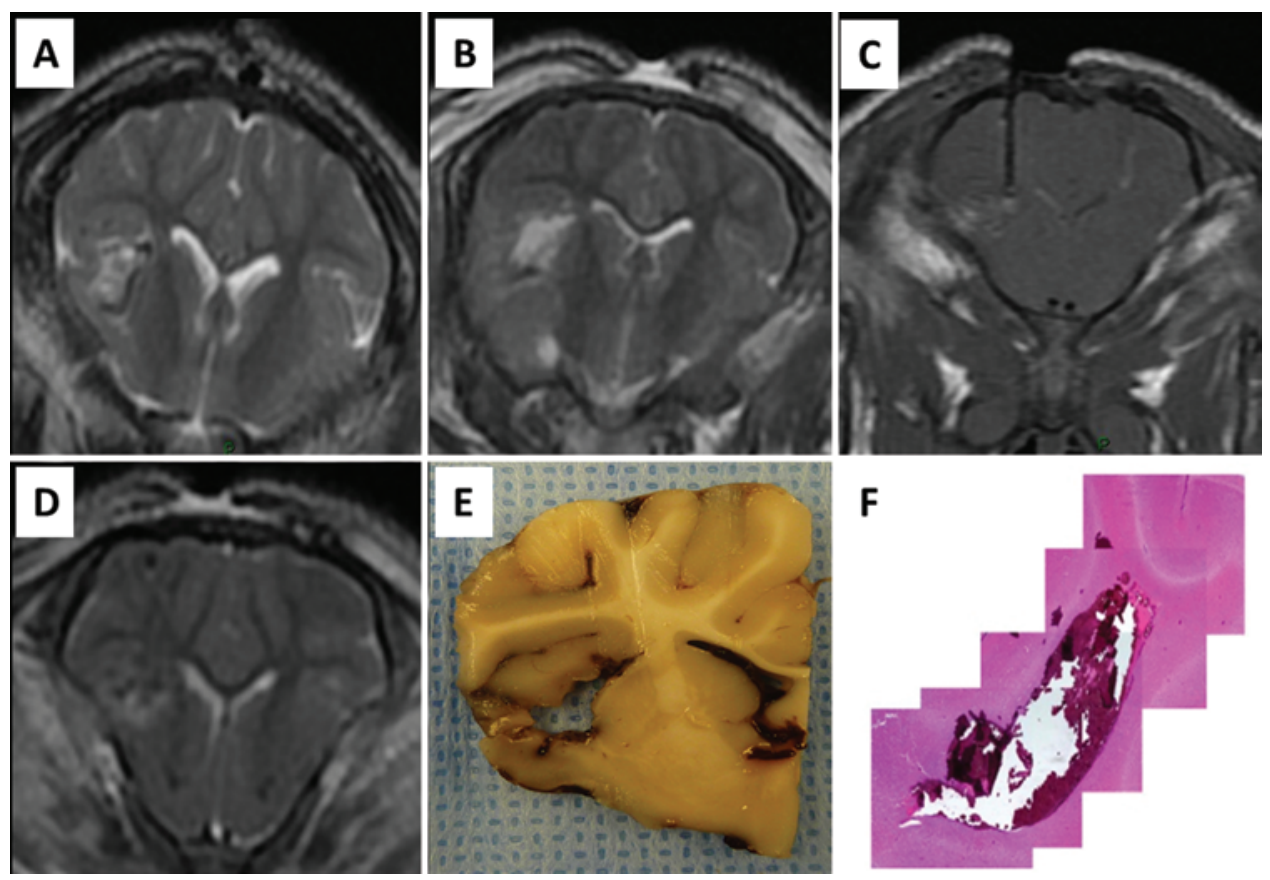

FIG. 5. Transcranial MRgFUS for ICH in swine. A: Pretreatment image of the ICH showing a dark mixed T2-weighted signal. B: Post-MRgFUS image demonstrating complete sonothrombolysis with increased T2 signal. C: MRI-guided aspiration of liquefied clot using an 18-gauge angiocatheter. D: Postaspiration image showing minimal residual ICH. E and F: Gross pathological and histological images demonstrating no edema or additional neuronal damage to the surrounding brain as a result of MRgFUS. H \& E, original magnification ×2 (F). Printed with permission from Monteith et al: J Neurosurg 118:1035-1045, 2013.

Acquisition of data: Monteith. Analysis and interpretation of data: Monteith, Harnof. Drafting the article: Monteith, Harnof. Critically revising the article: all authors. Reviewed submitted version of manuscript: all authors. Approved the final version of the manuscript on behalf of all authors: Monteith.

\section{References}

1. Broderick JP, Brott TG, Duldner JE, Tomsick T, Huster G: Volume of intracerebral hemorrhage. A powerful and easy-touse predictor of 30-day mortality. Stroke 24:987-993, 1993

2. Brott T, Broderick J, Kothari R, Barsan W, Tomsick T, Sauerbeck L, et al: Early hemorrhage growth in patients with intracerebral hemorrhage. Stroke 28:1-5, 1997

3. Butcher KS, Baird T, MacGregor L, Desmond P, Tress B, Davis $\mathrm{S}$ : Perihematomal edema in primary intracerebral hemorrhage is plasma derived. Stroke 35:1879-1885, 2004

4. Dey M, Stadnik A, Awad IA: Thrombolytic evacuation of intracerebral and intraventricular hemorrhage. Curr Cardiol Rep 14:754-760, 2012

5. Diamond SL, Anand S: Inner clot diffusion and permeation during fibrinolysis. Biophys J 65:2622-2643, 1993

6. Durst C, Monteith S, Sheehan J, Moldovan K, Snell J, Eames $\mathrm{M}$, et al: Optimal imaging of in vitro clot sonothrombolysis by MR-guided focused ultrasound. J Neuroimaging [epub ahead of print], 2011

7. Elias J, Huss D, Loomba J, Khaled M, Frysinger R, Sperling S, et al: A phase 1 study of MR-guided focused ultrasound thalamotomy for the treatment of medication-refractory essential tremor. Presented at the Current and Future Application of Focused Ultrasound 2012, 3rd International Symposium. Washington, DC, 2012 (Abstract) (http://www.fusfoundation. org/media/FUSF_Symposium_2012_Program_WebVersion. pdf) [Accessed March 28, 2013]
8. Fatar M, Stroick M, Griebe M, Alonso A, Hennerici MG, Daffertshofer M: Brain temperature during $340-\mathrm{kHz}$ pulsed ultrasound insonation: a safety study for sonothrombolysis. Stroke 37:1883-1887, 2006

9. Francis CW, Blinc A, Lee S, Cox C: Ultrasound accelerates transport of recombinant tissue plasminogen activator into clots. Ultrasound Med Biol 21:419-424, 1995

10. Gebel JM Jr, Jauch EC, Brott TG, Khoury J, Sauerbeck L, Salisbury S, et al: Natural history of perihematomal edema in patients with hyperacute spontaneous intracerebral hemorrhage. Stroke 33:2631-2635, 2002

11. Gebel JM Jr, Jauch EC, Brott TG, Khoury J, Sauerbeck L, Salisbury S, et al: Relative edema volume is a predictor of outcome in patients with hyperacute spontaneous intracerebral hemorrhage. Stroke 33:2636-2641, 2002

12. Guthkelch AN, Carter LP, Cassady JR, Hynynen KH, Iacono RP, Johnson PC, et al: Treatment of malignant brain tumors with focused ultrasound hyperthermia and radiation: results of a phase I trial. J Neurooncol 10:271-284, 1991

13. Hynynen K: MRI-guided focused ultrasound treatments. Ultrasonics 50:221-229, 2010

14. Hynynen K, Jolesz FA: Demonstration of potential noninvasive ultrasound brain therapy through an intact skull. Ultrasound Med Biol 24:275-283, 1998

15. Inaji M, Tomita H, Tone O, Tamaki M, Suzuki R, Ohno K: Chronological changes of perihematomal edema of human intracerebral hematoma. Acta Neurochir Suppl 86:445-448, 2003

16. Jagannathan J, Sanghvi NT, Crum LA, Yen CP, Medel R, Dumont AS, et al: High-intensity focused ultrasound surgery of the brain: part 1-A historical perspective with modern applications. Neurosurgery 64:201-211, 2009

17. Jeanmonod D, Moser D, Magara A, Kowalski M, Bühler R, Pourtehrani P, et al: Study on incisionless transcranial magnetic resonance-guided focused ultrasound treatment of Par- 
kinson's disease: safety, accuracy and initial clinical outcomes. Presented at the Current and Future Application of Focused Ultrasound 2012, 3rd International Symposium. Washington, DC, 2012 (Abstract) (http://www.fusfoundation.org/media/ FUSF_Symposium_2012_Program_WebVersion.pdf) [Accessed March 25, 2013]

18. Jeanmonod D, Werner B, Morel A, Michels L, Zadicario E, Schiff G, et al: Transcranial magnetic resonance imagingguided focused ultrasound: noninvasive central lateral thalamotomy for chronic neuropathic pain. Neurosurg Focus 32(1):E1, 2012

19. Kazui S, Naritomi H, Yamamoto H, Sawada T, Yamaguchi T: Enlargement of spontaneous intracerebral hemorrhage. Incidence and time course. Stroke 27:1783-1787, 1996

20. Labovitz DL, Halim A, Boden-Albala B, Hauser WA, Sacco RL: The incidence of deep and lobar intracerebral hemorrhage in whites, blacks, and Hispanics. Neurology 65:518-522, 2005

21. Martin E, Jeanmonod D, Morel A, Zadicario E, Werner B: High-intensity focused ultrasound for noninvasive functional neurosurgery. Ann Neurol 66:858-861, 2009

22. McDannold N, Arvanitis CD, Vykhodtseva N, Livingstone MS: Temporary disruption of the blood-brain barrier by use of ultrasound and microbubbles: safety and efficacy evaluation in rhesus macaques. Cancer Res 72:3652-3663, 2012

23. Medel R, Monteith SJ, Elias WJ, Eames M, Snell J, Sheehan JP, et al: Magnetic resonance-guided focused ultrasound surgery: Part 2: A review of current and future applications. Neurosurgery 71:755-763, 2012

24. Mendelow AD, Gregson BA, Fernandes HM, Murray GD, Teasdale GM, Hope DT, et al: Early surgery versus initial conservative treatment in patients with spontaneous supratentorial intracerebral haematomas in the International Surgical Trial in Intracerebral Haemorrhage (STICH): a randomised trial. Lancet 365:387-397, 2005

25. Mendelow AD, Unterberg A: Surgical treatment of intracerebral haemorrhage. Curr Opin Crit Care 13:169-174, 2007

26. Mohadjer M, Eggert R, May J, Mayfrank L: CT-guided stereotactic fibrinolysis of spontaneous and hypertensive cerebellar hemorrhage: long-term results. J Neurosurg 73:217-222, 1990

27. Monteith S, Harnof S, Wintermark M, Kassell N, Snell J, Eames M, et al: Transcranial MR guided focused ultrasound treatment of intracerebral hemorrhage. Presented at the Joint EANS Annual Meeting 4th World ICH Conference. Newcastle Upon Tyne, England, 2011 (Abstract) (http:// www.abstractserver.com/eansich2011/planner/index. php?go=abstract\&action $=$ abstract_iplanner\&absno $=116 \&$ EANSICH2011=0jigf8ce7f28j6m2m0th2ogfb0\&EANSICH20 11=0jigf8ce7f28j6m2m0th2ogfb0) [Accessed March 28, 2013]

28. Monteith S, Sheehan J, Medel R, Wintermark M, Eames M, Snell J, et al: Potential intracranial applications of magnetic resonance-guided focused ultrasound surgery. A review. J Neurosurg 118:215-221, 2013

29. Monteith SJ, Harnof S, Medel R, Popp B, Wintermark M, Lopes MBS, et al: Minimally invasive treatment of intracerebral hemorrhage with magnetic resonance-guided focused ultrasound. Laboratory investigation. J Neurosurg 118:10351045,2013

30. Morgan T, Awad I, Keyl P, Lane K, Hanley D: Preliminary report of the clot lysis evaluating accelerated resolution of intraventricular hemorrhage (CLEAR-IVH) clinical trial. Acta Neurochir Suppl 105:217-220, 2008

31. Morgan T, Zuccarello M, Narayan R, Keyl P, Lane K, Hanley D: Preliminary findings of the minimally-invasive surgery plus rtPA for intracerebral hemorrhage evacuation (MISTIE) clinical trial. Acta Neurochir Suppl 105:147-151, 2008

32. Moser D, Zadicario E, Schiff G, Jeanmonod D: Measurement of targeting accuracy in focused ultrasound functional neurosurgery. Neurosurg Focus 32(1):E2, 2012

33. Newell DW, Shah MM, Wilcox R, Hansmann DR, Melnychuk E, Muschelli J, et al: Minimally invasive evacuation of spontaneous intracerebral hemorrhage using sonothrombolysis. Clinical article. J Neurosurg 115:592-601, 2011

34. Qureshi AI, Mendelow AD, Hanley DF: Intracerebral haemorrhage. Lancet 373:1632-1644, 2009

35. Qureshi AI, Tuhrim S, Broderick JP, Batjer HH, Hondo H, Hanley DF: Spontaneous intracerebral hemorrhage. N Engl J Med 344:1450-1460, 2001

36. Ram Z, Cohen ZR, Harnof S, Tal S, Faibel M, Nass D, et al: Magnetic resonance imaging-guided, high-intensity focused ultrasound for brain tumor therapy. Neurosurgery 59:949956, 2006

37. Rohde V, Rohde I, Thiex R, Ince A, Jung A, Dückers G, et al: Fibrinolysis therapy achieved with tissue plasminogen activator and aspiration of the liquefied clot after experimental intracerebral hemorrhage: rapid reduction in hematoma volume but intensification of delayed edema formation. J Neurosurg 97: 954-962, 2002

38. Rosenschein U, Furman V, Kerner E, Fabian I, Bernheim J, Eshel Y: Ultrasound imaging-guided noninvasive ultrasound thrombolysis: preclinical results. Circulation 102:238-245, 2000

39. Sakharov DV, Hekkenberg RT, Rijken DC: Acceleration of fibrinolysis by high-frequency ultrasound: the contribution of acoustic streaming and temperature rise. Thromb Res 100: 333-340, 2000

40. Sudlow CL, Warlow CP: Comparable studies of the incidence of stroke and its pathological types: results from an international collaboration. Stroke 28:491-499, 1997

41. Sun J, Hynynen K: Focusing of therapeutic ultrasound through a human skull: a numerical study. J Acoust Soc Am 104: $1705-1715,1998$

42. Treat LH, McDannold N, Zhang Y, Vykhodtseva N, Hynynen $\mathrm{K}$ : Improved anti-tumor effect of liposomal doxorubicin after targeted blood-brain barrier disruption by MRI-guided focused ultrasound in rat glioma. Ultrasound Med Biol 38: 1716-1725, 2012

Manuscript submitted January 11, 2013.

Accepted February 19, 2013.

Please include this information when citing this paper: DOI: 10.3171/2013.2.FOCUS1313.

Address correspondence to: Stephen Monteith, M.D., Department of Neurosurgery, University of Virginia Health System, P.O. Box 800212, Charlottesville, Virginia 22908. email: stephen.monteith@ gmail.com. 